\title{
A UTILIZAÇÃO DAS TERAPIAS COMPLEMENTARES NOS CUIDADOS PALIATIVOS: BENEFÍCIOS E FINALIDADES*
}

Juliana Souza Caires', Tuanny Argolo de Andrade1, Juliana Bezerra do Amaral², Maria Thais de Andrade Calasans ${ }^{3}$, Michelle Daiane da Silva Rocha ${ }^{4}$

${ }^{1}$ Enfermeira. Escola Bahiana de Medicina e Saúde Pública. Lauro de Freitas-BA-Brasil.

${ }^{2}$ Enfermeira. Doutora em Enfermagem. Universidade Federal da Bahia. Salvador-BA-Brasil.

${ }^{3}$ Enfermeira. Mestre em Enfermagem. Escola Bahiana de Medicina e Saúde Pública. Salvador-BA-Brasil.

${ }^{4}$ Enfermeira. Residência em Oncologia. Instituto Nacional de Câncer. Salvador-BA-Brasil.

RESUMO: Estudo exploratório-descritivo que foi parte de recorte de um projeto de pesquisa realizado em 2011 e 2012, teve por objetivo analisar a utilização das terapias complementares nos cuidados paliativos pelas instituições brasileiras credenciadas nas Associações Nacionais e Latino-Americana de Cuidados Paliativos. A coleta de dados, com seis representantes de serviços de saúde, se deu por meio de um formulário semiestruturado. Os dados foram analisados quantitativamente utilizando-se percentagem simples e, qualitativamente, apoiou-se na Análise de Conteúdo. Os resultados revelaram que a musicoterapia, acupuntura e massagem foram as modalidades mais utilizadas nos cuidados paliativos; as terapias complementares, aliadas ao tratamento convencional, ajudam a aliviar a ansiedade, a depressão e a dor dos pacientes, promovendo relaxamento e facilitando a relação e a interação entre profissional-paciente-família. Assim, o uso das terapias complementares nos cuidados paliativos deve ser valorizado e empregado pelos profissionais habilitados, uma vez que colabora para melhora da qualidade de vida. DESCRITORES: Cuidados paliativos; Terapias complementares; Enfermagem.

\section{THE USE OF COMPLEMENTARY THERAPIES IN PALLIATIVE CARE: BENEFITS AND PURPOSES}

\begin{abstract}
This exploratory-descriptive study was an excerpt from the research project undertaken in 2011 to 2012, and aimed to analyze the use of complementary therapies in palliative care by Brazilian institutions accredited with the Brazilian and Latin-American Associations of Palliative Care. Data collection, with six representatives of the health services, occurred through a semi-structured questionnaire. The data were analyzed quantitatively using simple percentages, and qualitatively, based in Content Analysis. The results revealed that music therapy, acupuncture and massage were the modalities used most in palliative care; the complementary therapies, allied with conventional treatment, help to relieve the patients' anxiety, depression and pain, promoting relaxation, and facilitating the relationship with, and the interaction between, professional-patient-family. Thus, the use of the complementary therapies in palliative care must be valued and used by trained professionals, as it contributes to improving quality of life.
\end{abstract}

DESCRIPTORS: Palliative care; Complementary therapy; Nursing.

\section{LA UTILIZACIÓN DE LAS TERAPIAS COMPLEMENTARIAS EN LOS CUIDADOS PALIATIVOS: BENEFICIOS Y FINALIDADES}

RESUMEN: Estudio exploratório descriptivo que hizo parte de recorte de un proyecto de investigación realizado en 2011 y 2012 cuyo objetivo fue analizar la utilización de terapias complementarias en los cuidados paliativos por las instituciones brasileñas habilitadas en las Asociaciones Nacionales y Latinoamericanas de Cuidados Paliativos. Los datos fueron obtenidos con seis representantes de servicios de salud por medio de formulario semiestructurado. Fueron analizados de modo cuantitativo utilizándose porcentaje simple. Cualitativamente, se apoyó en el Análisis de Contenido. Los resultados revelaron que la musicoterapia, acupuntura y masaje fueron las modalidades más utilizadas en los cuidados paliativos; las terapias complementares, aliadas al tratamiento convencional, ayudan a aliviar la ansiedad y la depresión y el dolor de los pacientes, promoviendo relajamiento y facilitando la relación y la interacción entre profesional-paciente-familia. De ese modo, el uso de las terapias complementares en los cuidados paliativos debe ser valorado y empleado por los profesionales habilitados, una vez que colabora para mejorar la cualidad de vida. DESCRIPTORES: Cuidados paliativos; Terapias complementares; Enfermería.

*Estraído do Projeto de Iniciação Científica financiado pela Fundação de Amparo à Pesquisa do Estado da Bahia.

Autor Correspondente:

Juliana Bezerra do Amaral

Universidade Federal da Bahia

Av. Dr. Augusto Viana, s/n - 40110-060 - Salvador-BA-Brasil

E-mail: julianabamaral@yahoo.com.br
Recebido: $14 / 10 / 2013$

Finalizado: $22 / 05 / 2014$ 


\section{INTRODUÇÃO}

Atualmente, o conceito de incurabilidade de uma doença não indica o final do tratamento, mas a necessidade, na abordagem, de mudança dos objetivos. Os princípios dos cuidados paliativos apresentam elementos fundamentais e ideais para atender a real demanda da população acometida, principalmente, por enfermidades crônicas e não transmissíveis ${ }^{(1)}$.

A Organização Mundial de Saúde (OMS) definiu os Cuidados Paliativos como

abordagem que promove a qualidade de vida de pacientes e de seus familiares, ao enfrentarem doenças que ameacem a continuidade da vida, por meio da prevenção e do alívio do sofrimento. Requer identificação precoce, avaliação e tratamento da dor e outros problemas de natureza física, psicossocial e espiritual. ${ }^{(2: 16)}$.

Desse modo, a OMS destaca, neste conceito atualizado, a participação da família e dos cuidados direcionados à multidimensionalidade da pessoa. Considerando a necessidade de um cuidado ativo e total, as terapias complementares são úteis nos cuidados paliativos, uma vez que visam prestar assistência integral ao indivíduo, contemplando os aspectos físicos, sociais, emocionais e espirituais.

O uso das terapias complementares é cada vez mais comum, as pessoas que enfrentam o desafio de doenças incuráveis são atraídas pela oportunidade de ter outras possibilidades de cuidados. Diante dessa perspectiva, a procura por essas terapêuticas visa auxiliar o tratamento convencional e melhorar a qualidade de vida. $\mathrm{O}$ emprego das mesmas não deve ser exclusivo nem excludente, mas integrador, por serem as terapias compatíveis com os princípios dos cuidados paliativos e por estarem relacionadas à autonomia do indivíduo ${ }^{(3)}$.

No final da década de 1970, no intuito de estimular a criação de políticas direcionadas às práticas complementares, a OMS instituiu o Programa de Medicina Tradicional. Por meio de informes e resoluções, divulgou o seu interesse em impulsionar os Estados-membros a legislarem políticas públicas para o uso racional e integrado da medicinal tradicional e complementar/ alternativa nos sistemas nacionais de atenção à saúde. Ao mesmo tempo, foi incentivada a realização de estudos científicos a fim de promover conhecimento sobre a segurança, eficácia e qualidade dessa terapia ${ }^{(4)}$.

Em 2003, no Brasil, iniciou-se a construção da Política Nacional de Práticas Integrativas e Complementares (PNPIC) com a finalidade de atender às diretrizes e indicações de várias Conferências Nacionais de Saúde e às recomendações da OMS. A PNPIC, no Sistema Único de Sáude (SUS), foi aprovada em fevereiro de 2006 e publicada na forma das Portarias Ministeriais n. 971 em 03 de maio de 2006 e n. 1.600, de 17 de julho de 2006 ${ }^{(4)}$.

A partir dessas observações; do cenário atual da saúde; do aumento cada vez mais significativo de pessoas com enfermidades incuráveis e progressivas, que demanda cuidado integral; da abordagem holística das terapias complementares e dos cuidados paliativos, identificou-se a importância desta pesquisa, com o objetivo analisar a utilização das terapias complementares nos cuidados paliativos, pelas instituições brasileiras credenciadas nas Associações Nacionais e Latino-Americana de Cuidados Paliativos.

\section{MÉTODO}

Pesquisa exploratória e descritiva com abordagem qualitativa e quantitativa, que teve como participantes instituições hospitalares, domiciliares, equipes de interconsulta e demais tipos de serviços de cuidados paliativos do território brasileiro que utilizam terapias complementares e que aceitaram participar da pesquisa. Este estudo é um recorte de um Projeto de Iniciação Científica fomentado pela Fundação de Amparo à Pesquisa do Estado da Bahia, intitulado "O Cenário Brasileiro da Utilização das Terapias Complementares nos Cuidados Paliativos", que foi realizado nos anos de 2011 e 2012.

Para identificação das instituições foi realizada busca dos serviços de cuidados paliativos cadastrados na Academia Nacional de Cuidados Paliativos, Associação Latino Americana de Cuidados Paliativos e Associação Brasileira de Cuidados Paliativos, por meio dos sites das referidas associações, sendo encontradas 86 instituições cadastradas. 
Das instituições identificadas, foi possível estabelecer contato com 73(84,9\%) serviços de saúde. Destas 56(76,7\%) utilizavam cuidados paliativos e $17(23,3 \%)$ não utilizavam, apesar de estarem cadastradas nas associações consultadas. Das 56(100\%) instituições com serviço de cuidados paliativos ativos apenas $14(25 \%)$ faziam uso de terapias complementares, estando localizadas 8(57,1\%) em São Paulo, 2(14,3\%) na Bahia e 1(7,1\%), nos Estados do Rio de Janeiro, Espírito Santo, Distrito Federal, e Alagoas.

A princípio, foi realizado um convite informal, por contato telefônico, com as instituições que faziam uso das terapias complementares nos cuidados paliativos. As mesmas foram convidadas a participar do estudo, sendo solicitado que um profissional que atuasse no serviço respondesse a um formulário. No entanto, 2(14,3\%) não aceitaram participar, 6(42,8\%) não responderam, mesmo após diversas tentativas de contato via correio eletrônico e telefônico e, por fim, $6(42,8 \%)$ responderam ao formulário da pesquisa. Dessa forma, somente essas foram incluídas nesta pesquisa, sendo 2(33,3\%) em São Paulo, 1(16,7\%) no Rio de Janeiro, 1(16,7\%) no Distrito Federal, $1(16,7 \%)$ em Alagoas e $1(16,7 \%)$ na Bahia.

Como instrumento de coleta dos dados foi utilizado um questionário semiestruturado com questões objetivas e subjetivas enviado por correio eletrônico, juntamente com o Termo de Consentimento Livre e Esclarecido e uma carta convite que identificava os pesquisadores, esclarecia o objetivo da pesquisa e ressaltava a participação voluntária no estudo. Considerou-se que a partir do momento em que o profissional respondesse ao e-mail, o mesmo estaria de acordo com a participação na pesquisa, não sendo obrigatório o retorno do termo assinado.

Os profissionais que responderam ao formulário foram 2(33,3\%) médicos, 2(33,3\%) enfermeiros, $1(16,7 \%)$ fisioterapeuta e $1(16,7 \%)$ administrador.

Os dados foram coletados após aprovação do Comitê de Ética da Escola Bahiana de Medicina e Saúde Pública, com o protocolo n. 254/2011, garantindo que todos os aspectos éticos ${ }^{(5)}$. Os dados quantitativos foram tabulados, tratados estatisticamente e apresentados por meio de percentagem simples, utilizando o programa Microsoft ${ }^{\circledR}$ Excel 2010; enquanto que os qualitativos foram analisados por meio da Análise de Conteúdo(6).
Com a construção do corpus foi possível realizar a leitura flutuante e exaustiva dos textos presentes nos questionários enviados, recortando e realizando uma síntese geral. Foram concebidas as ideias centrais com associação dos escritos mais relevantes em temas e, finalmente, a análise do material, organizado em categorias temáticas, com o referencial teórico relacionado ao tema.

\section{RESULTADOS}

Ao analisar as respostas dos seis participantes ao questionário da pesquisa, foi possível agrupar os achados em duas categorias: as terapias complementares utilizadas nos cuidados paliativos; e os benefícios e finalidades das terapias complementares nos cuidados paliativos.

\section{As Terapias Complementares Utilizadas nos Cuidados Paliativos}

Em relação às modalidades das terapias complementares utilizadas pelas instituições participantes, 14 foram citadas, destacandose: a musicoterapia, informada por $100 \%$ das instituições, seguida da acupuntura e massagem, por $67 \%$ (Tabela 1 ).

Tabela 1 - As Terapias Complementares utilizadas nos Cuidados Paliativos. Salvador-BA-Brasil, 2011-2012

\begin{tabular}{lcc}
\hline Terapias & $\mathbf{n}$ & $\mathbf{\%}$ \\
\hline Acupuntura & 4 & 67 \\
\hline Auricultura & 1 & 17 \\
\hline Cromoterapia & 1 & 17 \\
\hline Do-In & 1 & 17 \\
\hline Fitoterapia & 1 & 17 \\
\hline loga & 1 & 17 \\
\hline Massagem & 4 & 67 \\
\hline Meditação & 1 & 17 \\
\hline Musicoterapia & 6 & 100 \\
\hline Reflexologia & 1 & 17 \\
\hline Reiki & 3 & 50 \\
\hline Shiatsu & 1 & 17 \\
\hline Terapia da dança & 1 & 17 \\
\hline Toque terapêutico & 1 & 17 \\
\hline Visualização & 2 & 33 \\
\hline
\end{tabular}




\section{Os Benefícios e Finalidades das Terapias Complementares nos Cuidados Paliativos}

As principais finalidades do uso das terapias complementares, utilizadas pelas instituições participantes foram: complementação do tratamento clínico e o alivio dos sintomas, em especial destacaram a ansiedade (100\%), seguida da depressão e dor, ambas com 83\% (Tabela 2).

Tabela 2 - Sintomas aliviados com o uso das Terapias Complementares nos Cuidados Paliativos $(\mathrm{N}=6)$ Salvador-BA-Brasil, 2011-2012

\begin{tabular}{lcc}
\hline Sintomas & $\mathbf{n}$ & $\mathbf{\%}$ \\
\hline Ansiedade & 6 & 100 \\
\hline Constipação & 2 & 33 \\
\hline Depressão & 5 & 83 \\
\hline Diarreia & 1 & 17 \\
\hline Dispneia & 2 & 33 \\
\hline Dor & 5 & 83 \\
\hline Incontinência & 1 & 17 \\
\hline Insônia & 2 & 33 \\
\hline Náusea e Vômitos & 3 & 50 \\
\hline Pirose & 1 & 17 \\
\hline Soluço & 2 & 33 \\
\hline
\end{tabular}

Quanto aos benefícios da utilização das terapias complementares, nos pacientes sob cuidados paliativos, foi mencionado: promoção do relaxamento; oportunidade de contato com o paciente e estabelecimento de uma relação entre profissional - paciente. Essas terapias são usadas, também, para evitar o isolamento e a depressão; facilitar interação entre paciente e família; proporcionar melhora na qualidade de vida e potencializar o efeito dos medicamentos no controle da dor. A musicoterapia foi citada como auxiliadora no resgate e fraternização com familiares, promovendo momentos de prazer e relaxamento para os pacientes e seus familiares. Recorte de depoimentos dos entrevistados referem estas informações:

Elas potencializam o efeito dos medicamentos no controle da dor, auxiliaram no controle de vários sintomas, inclusive aqueles não relatados ao médico e, principalmente, proporcionaram melhora na qualidade de vida. (Questionário 1)
Promovem integração entre o paciente, cuidadores e equipe multidisciplinar. Aliviam a ansiedade, depressão e dor. (Questionário 2)

Promovem relaxamento, alívio da dor oncológica e, acima de tudo, a oportunidade de contato que o paciente recebe. Temos certeza de que é imprescindível nos Cuidados Paliativos que praticamos desde 1997. (Questionário 3)

Complementa o tratamento dos sintomas com raros efeitos adversos, e a musicoterapia, em especial, facilita interação entre paciente e família. (Questionário 4)

Proporciona relaxamento, um maior estreitamento na relação profissional- paciente, evitando o isolamento e a depressão tão comuns nesta fase. (Questionário 5)

A musicoterapia promove momentos de prazer e relaxamento para os pacientes e familiares. (Questionário 6)

\section{DISCUSSÃO}

Apesar de bastante incipiente, os cuidados paliativos no Brasil, comparados com outros países como Inglaterra, Estados Unidos e Argentina, vem apresentando paulatinamente um aumento quantitativo de unidades de saúde que estão utilizando os seus princípios ${ }^{(7)}$.

No que se refere ao uso das terapias complementares nos cuidados paliativos, verificase discretas publicações que incentivem a utilização das mesmas. Uma pesquisa realizada na cidade do Texas-EUA identificou que dos 110 hospices que participaram do inquérito, a maioria $(56,4 \%)$ oferecia algum tipo de terapia complementar aos seus clientes ${ }^{(8)}$. Outra investigação com 30 serviços italianos de cuidados paliativos revelou que todos praticavam pelo menos uma modalidade ${ }^{(9)}$.

As terapias complementares são consideradas terapias que promovem o alívio dos sintomas de ordem física, psicológica e emocional. A dor é a causa mais comum de sofrimento e incapacidade que afeta milhares de pessoas em todo mundo. É considerado o sintoma mais frequente em 
pacientes fora de possibilidades terapêutica de cura, o que ocasiona grande desconforto e interfere diretamente na sua qualidade de vida(10).

Neste estudo, as terapias complementares são utilizadas para o controle da dor e para a depressão por 5(83\%) serviços de cuidados paliativos. Houve resultado similar em uma revisão sistemática, a qual mostrou que a presença da dor, depressão, insônia e alterações gastrointestinais são as principais razões para indicação das terapias complementares na população em geral(11). Igualmente, os resultados revelaram, ainda, que as terapias complementares são utilizadas para o alívio de sintomas como insônia, constipação, soluço e pirose por 2(33\%) instituições participantes ${ }^{(11)}$.

Corroborando com esses dados, um estudo demonstrou que as condições mais comuns que levam os médicos clínicos a utilizarem as terapias complementares nos pacientes sob cuidados paliativos são: síndromes dolorosas, condições psicológicas e doenças crônicas em geral ${ }^{(12)}$.

Além da dor outros sintomas como fadiga, dispneia, alterações cognitivas, perda de apetite, caquexia, náusea e depressão, se apresentam concomitantemente, causando intenso sofrimento, incapacidade e prejuízo à qualidade de vida. A atenção a todos esses sintomas é imprescindível quando se trata de cuidados paliativos ${ }^{(13)}$.

A prevalência da fadiga pode chegar a $95 \%$ nas pessoas com câncer ${ }^{(14)}$. Contudo os serviços participantes não informaram, de forma específica a utilização das terapias complementares para alívio deste sintoma de grande impacto para o bem estar do paciente. Possivelmente uma das razões seja o escasso diagnóstico e tratamento da fadiga oferecido pelos profissionais médicos ${ }^{(14)}$.

As náuseas e os vômitos também são sintomas preponderantes nos pacientes sob cuidados paliativos, podendo ocasionar alterações psicofisiológicas $^{(15-16)}$. Em uma investigação sobre a utilização de hipodermóclise em pacientes sob cuidados paliativos, as náuseas e vômito estiveram presentes em mais de $50 \%$ dos pacientes ${ }^{(16)}$.

Em consonância com algumas investigações ${ }^{(11,16)}$, os participantes relataram que as terapias complementares podem ajudar a lidar com o estresse, a melhorar o humor, a dar-lhes um maior senso de controle e a diminuir os desconfortos associados à doença e ao tratamento. Além disso, as terapias possuem o potencial de reduzir os efeitos adversos das drogas utilizadas no tratamento. Essa modalidade de tratamento não fornece, necessariamente, uma "cura para tudo", mas promove sensação de bem estar, o que contribui para uma experiência de estar mais em sintonia com a vida.

Outro sintoma indicado por 33\% dos participantes foi a dispneia que pode ser aliviada por meio do uso das terapias complementares. Estudo mostra que ela acomete cerca de 21 a 90\% dos pacientes com câncer, com ou sem envolvimento pulmonar ${ }^{(17)}$.

A musicoterapia foi referenciada por todas as instituições participantes (100\%). Alguns autores vêm destacando a importância da musicoterapia nos cuidados paliativos devido a seus diversos efeitos benéficos ${ }^{(1,18)}$. O simples ato de ouvir música pode produzir mudanças positivas no humor, restaurar a paz e o equilíbrio emocional, potencializar a expressividade emocional do ser, promover o relaxamento e a expressão dos sentimentos, tais como: tristeza, raiva e luto. A música também constitui um recurso de comunicação, que pode estabelecer a relação interpessoal, ajudar a retomar os sentimentos e lembranças do passado, proporcionando, assim, bem estar e conforto ao enfermo e aos seus cuidadores/familiares.

A música age sobre o sistema nervoso autônomo, atuando como estímulo na competição com a dor, fazendo com que a pessoa se distraia e desvie sua atenção. Desta forma, o estímulo doloroso é modulado, reduzindo o consumo de analgésicos e aliviando a tensão e o estresse, podendo, ainda, conduzir a momentos de alegria e relaxamento ${ }^{(3)}$. Acredita-se que a escolha principal dessa terapia pode estar relacionada ao baixo custo, maior disponibilidade de publicações científicas que comprovam os efeitos benéficos e à fácil acessibilidade.

Um estudo randomizado controlado com 138 pacientes com câncer analisou que a utilização da massagem associada à acupuntura e cuidados básicos no pós operatório, reduzem as queixas álgicas e o humor depressivo ${ }^{(19)}$. A massagem e a acunpuntura também foi citada por 4 (67\%) participantes para controle da dor e depressão.

Destaca-se, ainda, a importância da existência de profissionais habilitados para realizar as terapias complementares. A equipe de enfermagem, por permanecer mais tempo em contato com o 
paciente e seus familiares, pode sugerir a sua utilização no cotidiano do cuidado paliativo, uma vez que, conforme relatado, as mesmas possibilitam um estreitamento na relação profissional-paciente (Questionário 5).

A afinidade entre o terapeuta e o paciente, durante o uso das terapias complementares, bem como a presença intencional e autêntica deste profissional podem trazer grandes benefícios para o bem estar da pessoa a ser cuidada(20). As respostas dos pacientes ao utilizarem essas terapias foram facilitadas pelas interações com os terapeutas que contribuíram não apenas para o modelo de cuidar, mas também para as experiências dos pacientes de se sentirem seguros e cuidados. O jeito de cuidar e o emprego dessas terapias são proporcionados aos enfermos com o objetivo de aliviar o sofrimento da doença, permitir-lhes experimentar um renascimento nos aspectos psicossociais/espirituais/emocionais.

\section{CONSIDERAÇOES FINAIS}

O processo de identificação das instituições de cuidados paliativos no contexto nacional e o número de participantes foi uma limitação da pesquisa, considerando que nem todos os serviços estão cadastrados nas associações consultadas e dentre estes nem todos efetivamente se utilizam de terapias complementares.

No que se refere aos resultados, o estudo revelou que dentre as distintas modalidades de terapias complementares disponíveis, a musicoterapia, a massagem e a acupuntura são as mais utilizadas entre os participantes. As finalidades destacadas para o uso dessas terapêuticas são de complementar o tratamento convencional, principalmente, no controle de sintomas psicológicos, emocionais e físicos, como ansiedade, depressão e dor. Como benefício, evidenciou-se melhora da qualidade de vida com promoção do relaxamento e do prazer, bem como fortalecimento do vínculo entre paciente/ família/profissional.

Apesar da extrema importância identificada no uso das terapias complementares, parece-nos que essa terapêutica ainda é pouco inserida no contexto da assistência prestada ao paciente sob cuidados paliativos, nas instituições brasileiras de saúde.
Finalmente, o estudo contribui para o reconhecimento do potencial e legitimidade das terapias milenares no âmbito dos cuidados paliativos por divulgar as evidências, na práxis, dos benefícios das terapias complementares no cuidado no final de vida.

\section{REFERÊNCIAS}

1. Seki NH, Galheigo SM. O uso da música nos cuidados paliativos: humanizando o cuidado e facilitando o adeus. Interface comun. saúde educ. [Internet] 2010;14(33) [acesso em 06 de junho de 2013] Disponível: http://dx.doi.org/10.1590/S141432832010000200004

2. Academia Nacional de Cuidados Paliativos. Manual de cuidados paliativos. Rio de Janeiro: Diagraphic; 2009.

3. Amaral JB, Silva MJP. Práticas Complementares e os cuidados paliativos. In: Silva RS, Amaral JB, Malagutti W (organizadores). Enfermagem em cuidados paliativos: cuidando para uma boa morte. São Paulo: Martinari; 2013. p. 307-34.

4. Ministério da Saúde (BR). Política Nacional de Práticas Integrativas e Complementares no SUS - PNPIC-SUS. Brasília; 2006.

5. Ministério da Saúde (BR). Conselho Nacional de Saúde. Diretrizes e normas regulamentadoras de pesquisa envolvendo seres humanos. Resolução n. 196, de 10 de outubro de 1996. Brasília; 1996.

6. Bardin L. Análise de Conteúdo. Lisboa: Edições 70; 2009.

7. Silva RS, Amaral JB. Trajetória histórica do movimento hospice moderno e as contribuições de uma enfermeira. In: Silva RS, Amaral JB, Malagutti W (organizadores). Enfermagem em cuidados paliativos: cuidando para uma boa morte. São Paulo: Martinari; 2013. p. 37-47.

8. Olotu BS, Brown CM, Lawson KA, Barner JC. Complementary and Alternative Medicine Utilization in Texas Hospices: Prevalence, Importance, and Challenges. Am J Hosp Palliat Care. 2014;31(3):254-9.

9. Belletti M, Mallia L, Lucidi F, Reichmann S, Mastroianni C, De Marinis MG, et al. Complementary therapy and support services for formal and informal caregivers in Italian palliative care hospices: an exploratory and descriptive study. Support Care Cancer. 2011;19(12):1939-47

10. Amaral JB, Calasans MTA. As dimensões da dor na pessoa sob cuidados paliativos. In: Silva RS, Amaral JB, Malagutti W (organizadores). Enfermagem em 
cuidados paliativos: cuidando para uma boa morte. São Paulo: Martinari; 2013. p. 149-64.

11. Frass $M$, Strassl RP, Friehs $H$, Mullner $M$, Kundi $M$, Kaye A. Use and acceptance of complementary and alternative medicine among the general population and medical personnel: a systematic review. Ochsner J. 2012;12(1):45-56

12. Giannelli $M$, Cuttini $M$, Da Frè $M$, Buiatti E. General practitioners' knowledge and practice of complementary/alternative medicine and its relationship $\mathrm{p}$ with life-styles: a population-based survey in Italy. Bio Med Central. 2007;8(30).

13. Pimenta CAM, Mota DDCF. Educação em cuidados paliativos: componentes essenciais. In: Pimenta CAM, Mota DDCF, Cruz DALM. Dor e cuidados paliativos. São Paulo: Manole; 2006. p. 29-44.

14. Chiba T, Cabral LRB. Fadiga, sudorese e prurido. In: Academia Nacional de Cuidados Paliativos. Manual de cuidados paliativos. Rio de Janeiro: Diagraphic; 2009. p. 132-8.

15. Maciel MGS, Bettega R. Náusea e vômito. In: Academia Nacional de Cuidados Paliativos. Manual de cuidados paliativos. Rio de Janeiro: Diagraphic; 2009. p. 117-23.

16. Justino ET, Tuoto FS, Kalinke LP, Mantovani MF. Hipodermóclise em pacientes oncológicos sob cuidados paliativos. Cogitare enferm. [Internet] 2013;18(1) [acesso em 06 jun 2013]. Disponível: http://ojs.c3sl.ufpr.br/ojs/index.php/cogitare/article/ view/31307/20018

17. Carvalho RT. Dispneia, tosse e hipersecreção de vias aéreas. In: Academia Nacional de Cuidados Paliativos. Manual de cuidados paliativos. Rio de Janeiro: Diagraphic; 2009. p. 104-16.

18. Sales CA, Silva VA, Pilger C, Marcon SS. A música na terminalidade humana: concepções dos familiares. Rev Esc Enferm USP. [Internet] 2011;45(1) [acesso em 17 jul 2013]. Disponível: http://dx.doi.org/10.1590/ S0080-62342011000100019

19. Mehling WE, Jacobs B, Acree M, Wilson L, Bostrom A, West J, et al. Symptom management with massage and acupuncture in postoperative cancer patients: a randomized controlled trial. J Pain Symptom Manage. 2007;33(3):258-66.

20. Nelson JP. Being in Tune With Life Complementary Therapy Use and Well-Being in Residential Hospice Residents. J. Holistic Nursing. 2006;24(3):152-61. 